\title{
Triaxial residual stresses in composite tube from austenitic stainless steel with welded ferritic steel cladding
}

\section{Yu.V. Taran ${ }^{1, *}$ J. Schreiber ${ }^{2}$, A.M. Balagurov ${ }^{1}$, U. Stuhr ${ }^{3}$, H. Kockelmann ${ }^{4}$, V.B. Zlokazov ${ }^{1}$}

${ }^{1}$ Frank Laboratory of Neutron Physics, JINR, Dubna, Russia

${ }^{2}$ Fraunhofer Institute for Nondestructive Testing (Dresden branch), Germany

${ }^{3}$ SINQ, Paul Scherrer Institute, Villigen, Switzerland

${ }^{4} \mathrm{MPA}$, Stuttgart University, Germany

* Contact author; e-mail: taran@nf.jinr.ru.

Keywords: neutron diffraction, residual stress, stainless steel, ferritic steel, cladding

\begin{abstract}
The triaxial residual stresses in a composite tube from an austenitic stainless steel with a welded ferritic steel cladding were measured by the time-of-flight neutron diffraction method on the POLDI instrument at the PSI SINQ neutron pulsed facility. The POLDI results are compared to the results obtained by the destructive turning out and ring core methods and theoretical predictions of calculations by the finite element method. Only for the tangential component of the stress tensor the semiquantitative agreement of the all used methods was observed. There was a clear discrepancy between the results of the different methods in the axial component. For the radial component all methods reveal quite small stresses, however, with some distinct differences in their distributions.
\end{abstract}

\section{Introduction}

Compared to carbon and alloy steels, all corrosion resistant alloys are expensive. In many cases, corrosion resistance is required only on the surface of the material and carbon or alloy steel can be clad with a more corrosion resistant alloy. Cladding can save up to $80 \%$ of the cost of using solid alloy. Cladding of carbon or low alloy steel can be accomplished in several ways including roll bonding, explosive bonding, weld overlaying and "wallpapering". Clad materials are widely used in chemical processes, offshore oil production, oil refining and electric power generation industries. Weld overlaying is commonly used to clad the surfaces of fabricated steel structures. However, the existence of uncontrollable residual stress distributions in welded materials prevents this method from being widely applied.

At ECRS-5 [1], we have presented the report about the measurements of the triaxial residual stresses in a composite tube from an austenitic stainless steel with a welded ferritic steel 
cladding by the time-of-flight neutron diffraction (ND) method on the ENGIN instrument at the ISIS neutron pulsed facility. Cladding by a weld overlaying of the ferritic steel on the austenitic tube is used to build compressive stresses on its outer surface and, as a result, to suppress stress corrosion. Investigations of the residual stresses in such composite tubes are important for developing of the optimal welding techniques.

However in this experiment, we have experienced difficulties with determination of a stress free reference to calculate the residual strains. Also some uncertainty in the residual strain determination was introduced due to absence of a strain state control at the last stage of cutting of the investigated sample from the tube. Nevertheless, we have observed a qualitative agreement of ND experimental difference of the radial and tangential stress tensor components with the data obtained by the destructive turning out method (TOM), the destructive ring core method (RCM), as well as those of the theoretical predictions of calculations by the finite element method (FEM). Details of the TOM and RCM measurements, as well as the FEM calculation are given in [2].

Taking into account these circumstances and the development in last years a new comb technique for stress free references (to our knowledge, a first application of this technique was done in [3]) we have repeated the time-of-flight ND experiment on the POLDI instrument at the PSI SINQ neutron pulsed facility with more well-formed geometry of a sample as well as with a comb sample machined by the electro-discharge method (EDM) from the same tube.

In this paper, the results of the last measurements of the residual stress state in the composite steel tube on the POLDI stress-diffractometer are presented. New results have allowed us to determine correctly the triaxial distribution of the residual stresses and compare them to the data obtained in the previous experiment and also to TOM, RCM and FEM results.

\section{Experimental details}

To fabricate the composite steel tube seven layers of ferritic steel 3NiMo 1UP were welded on a $15 \mathrm{~mm}$ thick tube from austenitic steel X6CrNiTi 1810 with an inner radius of $133 \mathrm{~mm}$. The outer radius of the manufactured tube was $168 \mathrm{~mm}$. Elastic constants and tensile strength were found to be equal to $\mathrm{E}=176 \mathrm{GPa}, \mathrm{v}=0.3, \mathrm{R}_{\mathrm{m}}=536 \mathrm{MPa}$ for austenite and $\mathrm{E}=205 \mathrm{GPa}$, $v=0.3, R_{m}=695 \mathrm{MPa}$ for ferrite. For measurements at neutron sources, the same samples were prepared. First, the $200 \mathrm{~mm}$ long tube was cut from the manufactured tube. Then a $70^{\circ}$ of arc circumference segment was truncated from this tube. With the extensometers placed in the middle of the segment oriented in the axial and tangential directions, the effect of stress release was measured at the inner and outer surfaces. This release has to be accounted for a recalculation of segment experimental data as applied to the whole tube. The sample investigated at ISIS facility [1] was cut out from the $70^{\circ}$ circumference segment in the form of a smaller segment. A small stress release was possible during the last stage of cutting but it was not controlled with any strain gages.

For the measurements on the POLDI stress-diffractometer, two small segments of $70^{\circ}$ arc and of $10 \mathrm{~mm}$ length in the axial direction were cut from the large segment. One of them was used as the main sample for measurements of the radial, axial and tangential components of the strain tensor. From another small segment, we have made the comb-sample of $10 \mathrm{~mm}$ 
thickness along the tangential direction. Teeth of $3 \times 10 \mathrm{~mm}^{2}$ cross-section and $8.5 \mathrm{~mm}$ height were machined by EDM. The width of slits between teeth was $0.3 \mathrm{~mm}$.

The POLDI instrument is the high intensity, high resolution time-of-flight stressdiffractometer at the SINQ neutron source. The gauge volume inside the sample was formed by a diaphragm in the primary neutron beam and the radial collimator with the space resolution of $2 \mathrm{~mm}$ in the scattering beam, respectively. For measurements of the radial and axial components a height and a width of the diaphragm were equal to 10 and $2 \mathrm{~mm}$, respectively, whereas the diaphragm height was decreased up to $2 \mathrm{~mm}$ to measure the tangential component. The initial processing of measured spectra was carried out using the in-house single peak fit program "Poldiausfit" which determined positions of the selected diffraction peaks in the d-spacing. Eight and seven resolved single peaks were observed in the austenite and ferrite phases, respectively. Using a set of the selected peak positions the lattice parameter for each spectrum was calculated by applying the autoindexing program "Autox" [4].

\section{Experimental results}

The autoindexing lattice parameters of the austenite and ferrite phases are presented in figure 1 for all three neutron scattering vector directions.
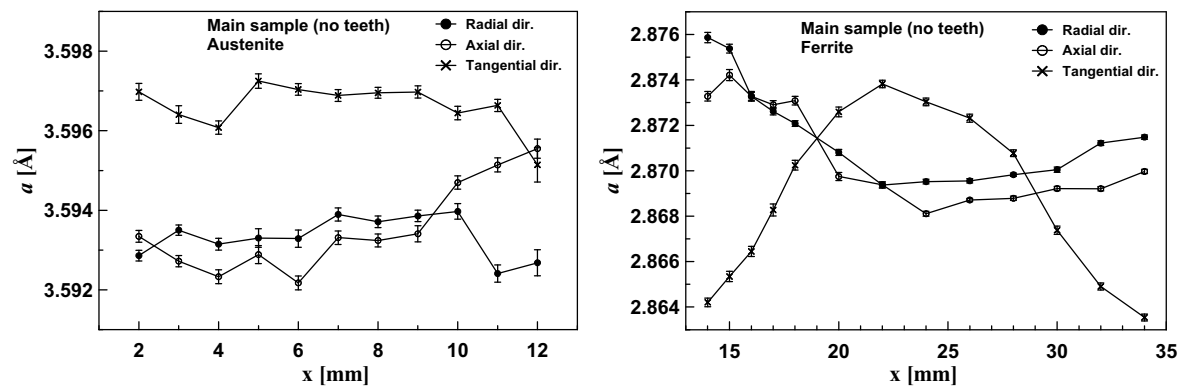

Figure 1. Radial, axial and tangential lattice parameters of the austenite (left) and ferrite (right) phases on the gauge volume coordinate $x$ ( $x=0$ and $35 \mathrm{~mm}$ correspond to the inner and outer edges of the composite tube, respectively).

To compare the ND results with the TOM, RCM and FEM results we have used a very effective approach based on calculation of the von Mises equivalent stress $\sigma_{e}$

$$
\sigma_{\mathrm{e}}=\sqrt{\frac{1}{2}\left[\left(\sigma_{\mathrm{rad}}-\sigma_{\mathrm{ax}}\right)^{2}+\left(\sigma_{\mathrm{rad}}-\sigma_{\mathrm{tan}}\right)^{2}+\left(\sigma_{\mathrm{ax}}-\sigma_{\mathrm{tan}}\right)^{2}\right]}
$$

that includes all possible pairwise stress/strain/lattice parameter differences

$$
\sigma_{i^{-}} \sigma_{j}=E\left(\varepsilon_{i}-\varepsilon_{j}\right) /(1+v)=\left(a_{i}-a_{j}\right) / a_{0}
$$

where $i$ and $j$ correspond to a pair of any scattering vector directions $(i \neq j)$. Note that the von Mises stress is independent of a hydrostatic stress component as well as of possible nonuniform redistribution of the chemical composition during the welding process, e.g., fixed carbon, and connected to it the stress free lattice parameter change along radius of the tube, 
especially, in the cladding. A low sensitivity of the von Mises stress to small deviations of $a_{0}$ from its true value is quite obviously out of equation (2). The results of the equivalent stress calculation are shown in figure 2. The qualitative agreement of the results of the all three used methods may be stated.

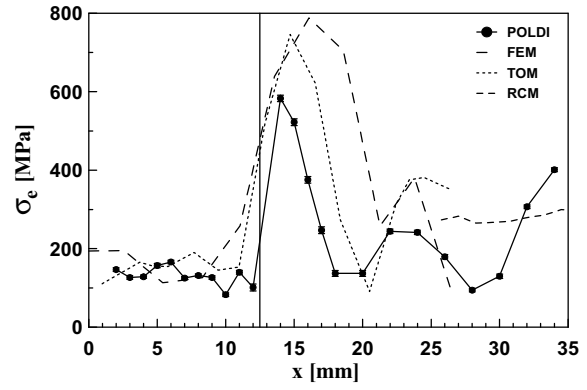

Figure 2. The von Mises stresses calculated from the POLDI, FEM, TOM and RCM results.

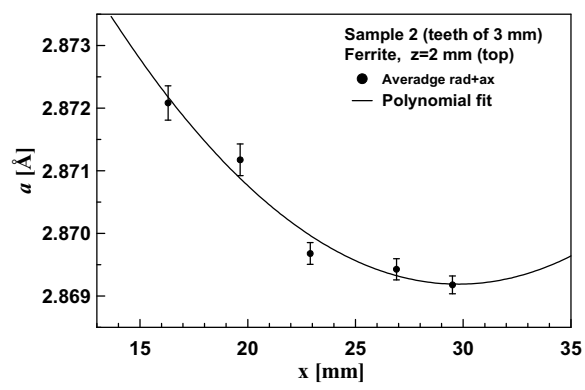

Figure 3. Average ferrite lattice parameters of the comb sample.

Absolute values of the stress tensor components from the POLDI data were calculated within the framework of the elastic model approximation:

$$
\sigma_{\mathrm{i}}=\frac{\mathbf{E}}{1+v}\left[\varepsilon_{\mathrm{i}}+\frac{v}{1-2 v}\left(\varepsilon_{\mathrm{rad}}+\varepsilon_{\mathrm{ax}}+\varepsilon_{\mathrm{tan}}\right)\right] .
$$

For this calculation it is critically important to use the best approximation of the parameter $a_{0}$ to a true stress free lattice parameter. As the stress free references we have used the lattice parameter radial dependence obtained by averaging of the comb sample results under the radial and axial directions and then by a polynomial fitting of the averaged experimental points (in figure 3, the results of averaging and fitting are shown). Unfortunately, the lattice parameter of the comb sample in the tangential direction was not measured for lack of the beam time. Therefore, we can not assert with certainty about a full stress relief in the comb sample. However, the elastic anisotropy analysis of the selected diffraction peaks is shown that, although, the dependence of the lattice parameter $a_{h k l}$ in the teeth tops of the both phases versus the anisotropy factor $G=\left(h^{2} k^{2}+h^{2} l^{2}+k^{2} l^{2}\right) /\left(h^{2}+k^{2}+l^{2}\right)^{2}$ had a noticeable scattering of the experimental points, on the whole, we can ascertain a practical absence of a slope in the anisotropy curves. This can point to the almost complete relief of the residual macrostresses in the comb sample. At that, the observed radial dependence of the ferrite lattice parameter in the comb sample (figure 3) can be put down to a variation of the chemical composition. The residual stress tensor components calculated in these assumptions are shown in figures 4-6 in comparison with the FEM and TOM results.

As the ND data were obtained for a small part of the tube they have to be corrected for the stress released during the cutting procedure. Only the axial and tangential components of the residual stresses were controlled during the sample cutting procedure. Assuming that the released stress varied linearly over the interval from the outer to the inner edge of the tube and that the radial component did not change essentially during cutting, the residual stresses in the uncut tube can be predicted from the ND data. The corrected ND results for the tangential component are shown in figure 6. 


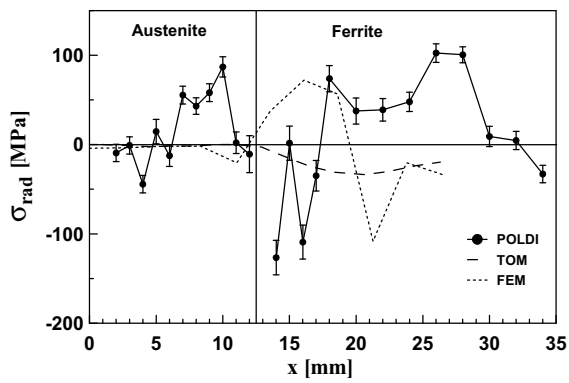

Figure 4. Residual stresses in the composite tube on the coordinate $x$ in the radial direction.

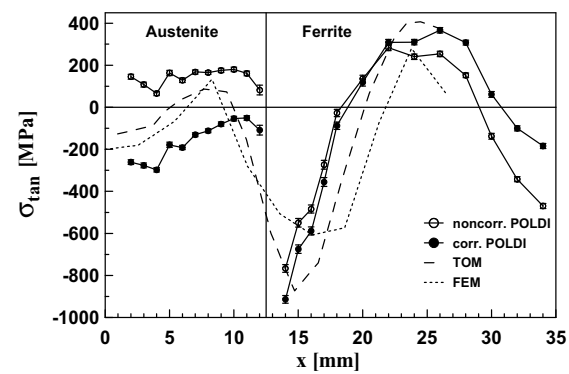

Figure 6. Residual stresses in the composite tube on the coordinate $x$ in the tangential direction.

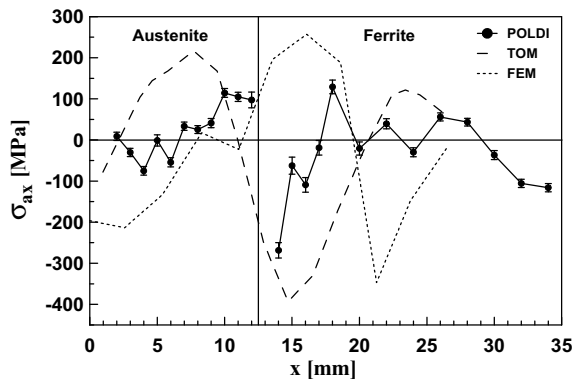

Figure 5. Residual stresses in the composite tube on the coordinate $x$ in the axial direction.

The results in the austenite phase had the greatest correction for the stress relief. But same correction has not introduced the principal changes in the ferrite phase. The ND results for the radial and axial directions were also corrected for the stress relief but the corrected results are not shown in figures 4-5 as corrections were insignificant.

Figures 4-5 demonstrates a contradictory picture between the POLDI and TOM experimental data and FEM calculated results for the radial and axial directions.

The presented methods have shown results for the radial direction completely contradicting each other. The less contradictory picture is observed for the axial direction. Note that the TOM and FEM curves for the axial direction are shifted onto about 5-6 $\mathrm{mm}$, at that, the peak values of the axial stresses are nearly equal. As to the radial stress component obtained by the TOM it was not directly determined, but calculated from a condition of the forces balance with use of the TOM results for two other stress components. Only for the tangential direction we can establish a semiquantitative agreement of the results of all presented methods, especially, for the ferritic phase. For the austenitic phase, some disagreement is certainly related to the uncontrollable influence of microstresses of the II type on the results of ND measurements. As it is visible from the figure the cladding produced the compressive tangential stress of about $800 \mathrm{MPa}$ close to the interface between the austenitic and ferritic parts of the composite tube.

The comparison of the curves in figures 4-6 clearly shows a reason of the qualitative agreement of results of the difference calculation of the radial and tangential stress components from the ENGIN results and the calculation of the von Mises equivalent stress from the POLDI results with the FEM and TOM results. In reality, the tangential stress component prevails over two others, namely, for it we have noticed the qualitative agreement of the all methods with each other. 


\section{Conclusion}

The present study has demonstrated the application of the time-of-flight neutron diffraction realized on the POLDI stress-diffractometer at the SINQ spallation source to measure the triaxial residual stresses in the composite tube from austenitic stainless steel with ferritic steel cladding fabricated by the welding overlaying technique. The objective of this research was to collect experimental information that can be helpful for the optimization of the welding technique.

The investigated sample was cut from the composite tube as a thin $70^{\circ}$ circumference segment by thickness of $10 \mathrm{~mm}$. The strain scanning was performed along the tube radius through the austenite and ferrite phases with three mutually perpendicular directions of the neutron scattering vector: radial, axial and tangential. The neutron diffraction pattern was analyzed by the single peak fit and autoindexing programs to determine a phase lattice parameter. The comb sample with teeth of $3 \mathrm{~mm}$ width machined by EDM from the tube was investigated to evaluate the stress free lattice parameter for the residual strain/stress calculations. Though only the radial and axial strain components were measured in the comb sample, the clear indication on the radial dependence of the stress free lattice parameter was obtained. We attribute this dependence to non-uniform redistribution of the chemical composition during the welding process, namely, fixed carbon.

Using the comb-sample lattice parameter results the triaxial residual stresses were revealed in the composite tube. The necessary correction was introduced into the axial and tangential stress components as only they were controlled during the sample cutting procedure. Comparison of the stress results from the ND measurements with the FEM, TOM and RCM results has shown that there was a semiquantitative agreement among all of the used methods only for the tangential direction. For two other stress components the contradictions between these methods were rather strong, especially, for a rather weak radial component.

The strong compressive tangential residual stress of about $800 \mathrm{MPa}$ was observed in the ferrite cladding close to the interface between the parent tube and the cladding. At that, the cladding has created smaller but quite sufficient tangential compressive stress in the austenite phase which can prevent stress corrosion of the tube in service.

\section{References}

1. Taran, Yu.V., Schreiber, J., Wright, J.S., 2000, Materials Science Forum, 347-349, 640.

2. Berreth, K. \& Kockelmann, H., 1995, Optimierung der Formschweißtechnik für druckführende und korrosiv beanspruchte Komponenten der chemischen Industrie. Abschlußbericht zum Forschungsvorhaben AiF-Nr. 8954 (MPA Universität Stuttgart), $41 \mathrm{p}$.

3. May, P.S., Wimpory, R.C., Webster, G.A., O'Down, N.P., 2000, REST Experimental Report no. 425, in The Annual Report 2000 of Studsvik Neutron Research Laboratory.

4. Zlokazov, V.B., 1995, Comp. Phys. Communications, 85, 415. 